\title{
Evaluación de trastornos de la personalidad en pacientes heroinómanos mediante el International Personality Disorders Examination (IPDE)
}

\author{
San Narciso, G.I.*; Gutiérrez, E.**; Sálz, P.A.***; González, Ma P.*** \\ BASCARÁN, M $\mathbf{M}^{\mathrm{a}} \mathbf{T}$ ***; BOBES, J.*** \\ *Clínica Asturias (Gijón). \\ **Unidad Tratamiento Toxicomanías - Mieres. Servicios Mentales del Principado de Asturias (SESPA). \\ ***Area de Psiquiatría. Facultad de Medicina. Universidad de Oviedo. \\ Enviar correspondencia a: \\ Area de Psiquiatría - Facultad de Medicina. Julián Clavería 6 - 3. 33006 Oviedo. Tfno: 98510 3552. FAX: 98 510 3552. E-mail: frank@correo.uniovi.e
}

\section{Resumen:}

Objetivos.- Determinar 1. Prevalencia de trastornos de la personalidad (TP) en pacientes adictos a opiáceos 2. Factores involucrados en la retención terapeútica a medio plazo 6 meses) 3. Indices de validez del módulo de screening del IPDE 4. Estabilidad diagnóstica a medio plazo del IPDE.

Pacientes.- 262 pacientes (135 procedentes de centro público y 127 de centro privado), que consecutivamente demandaron tratamiento entre septiembre de 1996 y mayo de 1998. Dichos pacientes fueron asignados a las siguientes modalidades terapeúticas: Programa de Mantenimiento con Metadona (PMM) en centro público $(n=53)$, Programa de Mantenimiento con Naltrexona (PMN) en centro público ( $n=$ 82) y $P M N$ en centro privado $(n=127)$.

Evaluación.- Versión Europea del Addiction Severity Index (EuropASI) e International Personality Disorders Examination (IPDE).

Resultados y conclusiones.- Estudio basal: mayor prevalencia de TP en los pacientes sometidos a PMM. Los a distribución de TP varía en función de la modalidad terapeútica, así en PMM los TP más prevalentes son el disocial, límite y paranoide, en PMN público el disocial, ansioso y límite y en PMN privado el límite, disocial e impulsivo. En líneas generales, los adictos a opiáceos que cumplen criterios IPDE de TP presentan una mayor gravedad de su adicción.

Estudio de seguimiento: el perfil de los pacientes que abandonan precozmente el tratamiento en PMN privado se caracteriza por: mayor dosis inicial de heroína consumida, mayor gravedad de la adicción (áreas de alcohol, drogas y estado psiquiátrico del EuropASI) y mayor número de diagnósticos IPDE de TP.

Estudio de validez del IPDE: El módulo de screening del IPDE ha demostrado elevada sensibilidad y moderada especificidad. EI IPDE se ha mostrado como un instrumento dotado de una adecuada estabilidad diagnóstica a medio plazo (6 meses).

Palabras Clave: Dependencia a opiáceos, Metadona, Naltrexona, Trastornos de la personalidad, IPDE

\section{Summary:}

Objectives: To determine - 1. The prevalence of personality disorders (PD) in opiate abusers 2. The factors involved in therapeutic retention over 6 months 3 . The validity indexes of the screening module of the IPDE 4 . The mean term diagnostic stability of the IPDE.

Patients: 262 patients (public sector: 135; private sector: 127) who consecutively sought treatment between September 1996 and May 1998 were evaluated. Patients were assigned to the following type of therapy: Methadone Maintenance Program (MMP) in a public center $(n=53)$, Naltrexone Maintenance Program (NMP) in a public center $(n=82)$, and NMP in a private center $(n=127)$.

Evaluation: European version of the Addiction Severity Index (EuropASI) and International Personality Disorders Examination (IPDE).

Results and Conclusions: Baseline: greater prevalence of $\mathrm{PD}$ in patients undergoing MMP. The distribution of PD varies according to the kind of therapy employed, the most prevalent PD in the MMP being antisocial, borderline, and paranoid; in the public NMP PDs were antisocial, avoidant, and borderline, and those of the private NMP borderline, antisocial, and impulsive. In general, opiate abusers who meet IPDE criteria for PD showed a greater severity of addiction.

Follow-up: the profile of patients who withdrew from treatment early in the private NMP group demonstrated a greater initial dosis of heroin, greater severity of addiction on the alcohol, drug, and psychiatric areas of the EuropASI, and a greater number of PDs according to the IPDE.

IPDE validity: The screening module of the IPDE showed high sensitivity and moderate specifity. The IPDE was demonstrated to be an instrument with good diagnostic stability over 6 months.

Key words: Opiate dependence, Methadone, Naltrexone, Personality disorders, IPDE 


\section{INTRODUCCION}

E I estudio de los trastornos de la personalidad (TP) lleva ímplicitas numerosas dificultades ya que existen numerosos aspectos (adecuación de criterios de definición, número de TP, agrupamiento de éstos, su clasificación -categorial versus dimensional...), que se cuestionan con tal intensidad que existen incluso investigadores que han llegado a considerar a estos problemas nosólogicos como insalvables, aconsejando una suspensión, al menos temporal, de los diagnósticos de TP dentro de la psiquiatría (Tyrer, 1988).

Estudios de comorbilidad psiquiátrica en pacientes adictos a opiáceos refieren elevadas tasas de TP en dichos pacientes. A pesar de que la prevalencia varía considerablemente de unos estudios a otros (del 25\% al $61 \%$ en los trabajos que utilizan el juicio clínico como criterio diagnóstico y del $56 \%$ al $100 \%$ en los que utilizan instrumentos estructurados para la recogida de datos y/o criterios diagnósticos DSM-III ó DSMIIIR -De Jong et al, 1993-), existe un importante porcentaje de pacientes que padece al menos un TP, no siendo nada desdeñable el número de ellos que están afectos de dos ó más TP (Nace et al, 1991; Weiss et al, 1993; Rutherford et al, 1994; Cervera et al, 1997; Gutiérrez et al, 1998b; San Narciso et al, 1998).

Las prevalencias de los distintos tipos de TP parecen variar en función de cuál sea la principal sustancia de abuso del paciente, del sexo y del estatus socioeconómico. En el caso de los pacientes dependientes de opiáceos, a pesar de que el TP antisocial es típicamente el más frecuentemente reportado, todo el espectro de TP ha sido involucrado en mayor o menor medida (Kosten et al, 1982; Rounsaville et al, 1982; Khantzian y Treece, 1985; Abbott et al, 1994; Rutherford et al, 1994; Cacciola et al, 1996; Cervera et al, 1997; Gutiérrez et al, 1998b; San Narciso et al, 1998).

La comorbilidad entre abuso de sustancias y TP tiene unas implicaciones claras. Así, los pacientes con diagnóstico de TP realizan un mayor consumo de drogas no habituales y presentan pautas peculiares en el uso-abuso de alcohol. En líneas generales, se encuentran menos satisfechos con su vida, son más impulsivos, están más aislados y muestran una mayor tendencia a padecer problemas afectivos (Bobes et al, 1990; Carey et al, 1991; Nace et al, 1991; Rousar et al, 1994; Rutherford et al, 1994; Brooner et al, 1997).

A pesar de que está ampliamente aceptado el hecho de que padecer un TP se asocia a una peor respuesta terapeútica (Kosten et al, 1989; Reich y Green, 1991; Nace y Davis, 1993), esta circunstancia no está totalmente consensuada (Alterman y Cacciola, 1991). En este sentido cabe comentar el estudio realizado por Pettinati et al (1991) donde dichos autores llevan a cabo un estudio de seguimiento de un año en una muestra de pacientes con dependencia a alcohol (un tercio de los mismos presentaba además abuso/dependencia a cocaína). Los pacientes diagnosticados de TP no presentaron mayores tasas de recaída que aquellos que carecían de dicho diagnóstico. No obstante, cuando se evaluaron los clusters específicos, los autores hallaron que los pacientes del cluster A (raros o excéntricos) y/o los del B (emocionales o erráticos) presentaban mayores tasas de recaídas que los del cluster $\mathrm{C}$ (ansiosos o temerosos) o que aquellos pacientes que no tenían ningún diagnóstico de TP.

Por otra parte, existen autores que han constatado que aquellos pacientes drogodependientes que sufren TP primario (no desencadenado por la propia toxicomanía), presentan una peor respuesta al tratamiento (McLellan, 1986; Gerstley et al, 1990; Randval y Vaglum, 1991a, b).

Por último, la existencia de TP también va a influir en el pronóstico, tanto por la diferente actitud como por la posibilidad de aparición de complicaciones en el curso de la toxicomanía (Kosten et al, 1989; Brooner et al, 1993; Perkins et al, 1993; Cacciola et al, 1996).

Otro punto que queremos señalar en este apartado de introducción, son las importantes limitaciones que actualmente existen tanto en los criterios diagnósticos como en los instrumentos de evaluación de los trastornos mentales. Es absolutamente necesario la creación de métodos estandarizados que sean útiles a la hora de reducir las aparentes discrepancias en las tasas de prevalencia de los distintos trastornos mentales y para conocer las necesidades de tratamiento de los mismos (Regier et al, 1998).

En este sentido, el International Personality Disorders Examination (IPDE) constituye una útil herramienta de evaluación de la personalidad, compatible con los sistemas de clasificación DSM-IV y CIE-10. El IPDE es un instrumento fruto de los esfuerzos realizados por la Wordl Health Organization (WHO) y de la Alcohol, Drug Abuse and Mental Health Administration (ADAMHA) para la obtención de instrumentos estandarizados de evaluación aplicables en todas las latitudes. Este instrumento cuenta con una estabilidad inter-examinador y estabilidad diagnóstica a lo largo del tiempo similar a la de otros instrumentos ampliamente utilizados en el diagnóstico de las psicosis, los trastornos afectivos, de ansiedad o de uso-abuso de sustancias (Loranger et al, 1994).

\section{OBJETIVOS}

En este trabajo se exponen resultados de tres estudios diferentes cada uno de los cuales perseguía unos objetivos específicos que se detallan a continuación. 


\section{Estudio de la muestra basal}

Pretende determinar la prevalencia de TP, su distribución según categorías diagnósticas y el perfil de gravedad asociado a el diagnóstico de TP, en pacientes adictos a opiáceos sometidos a distintas modalidades terapeúticas.

\section{Estudio de validez del Cuestionario de Evaluación IPDE}

Determinar los índices de validez del cuestionario de evaluación IPDE (módulo de screening del IPDE) en sus dos versiones DSM-IV y CIE-10.

\section{Estudio de seguimiento}

Determinar los factores involucrados a medio plazo en la retención terapeútica, así como determinar la estabilidad diagnóstica del IPDE.

\section{MATERIALY METODO}

\section{Pacientes}

La muestra utilizada en la realización de este estudio se ha recogido de dos fuentes diferentes (centro público y centro privado) de nuestro entorno.

Muestra procedente del centro público: Incluye 135 pacientes que consecutivamente demandaron tratamiento por consumo de opiáceos en la Unidad de Tratamiento de Toxicomanías de Mieres (Asturias) entre los meses de septiembre de 1996 y mayo de 1998.

Criterios de inclusión: diagnóstico DSM-IV de dependencia de opiáceos, ser mayor de 18 años, proceso nuevo o paciente dado de alta previamente (el período de tiempo para considerar que un paciente fue dado de alta se establece en 6 meses) y consentimiento informado por parte del paciente para su inclusión en el estudio.

Criterios de exclusión: politoxicómano cuya principal droga de consumo no sea un opiáceo, ser menor de 18 años, pacientes con enfermedad psiquiátrica que pudiera distorsionar la correcta evaluación y la validez de los cuestionarios (depresión severa, psicosis, retraso mental o deterioro cognitivo fueron descartados siguiendo criterios clínico-diagnósticos DSM-IV), falta de autorización del paciente.

Dichos pacientes una vez evaluados fueron asignados a un Programa de Mantenimiento con Metadona -PMM- (53 pacientes) o a un Programa de Mantenimiento con Naltrexona -PMN- (82 pacientes).
Muestra procedente del centro privado: Incluye 127 que acudieron solicitando tratamiento a la Clínica Médico Psicológica Asturias (Gijón), durante el período de tiempo comprendido entre septiembre de 1996 y mayo de 1998.

Criterios de inclusión: diagnóstico CIE-10 de dependencia a opiáceos, haber superado positivamente el tratamiento de desintoxicación (verificado mediante el test de naloxona y/o mediante analítica de opiáceos en orina) y consentimiento informado por parte del paciente.

Criterios de exclusión: La no superación del test de naloxona o la presentación de un urinoanálisis positivo a opiáceos, embarazo, lactancia o mujer fertil que no utilizara métodos fiables de anticoncepción, insuficiencia hepática y/o hepatitis aguda (transaminasas séricas iguales o superiores a 250 U.I.), antecedentes de hipersensibilidad a la naltrexona, presencia de enfermedades concomitantes que pudiesen interferir en el desarrollo del estudio y falta de autoriazación por parte del paciente.

Una vez valorado el caso y realizado el correspondiente reconocimiento físico y psíquico realizaron un tratamiento de desintoxicación para posteriormente ser incluidos en un PMN.

\section{Instrumentos de evaluación:}

- Versión europea del Addiction Severity Index EuropASI- (Kokkevi y Hartgers, 1995): Se trata de una entrevista semiestructurada diseñada para recoger de forma estandarizada información relevante sobre aspectos de la vida del paciente, que pudieran haber contribuido a su proceso de abuso-dependencia de alcohol y otras drogas.

Dicha entrevista explora las siguientes áreas: situación médica, empleo y soportes, uso de alcohol, uso de drogas, situación legal, relaciones familiares y sociales, estado psiquiátrico. En cada una de las áreas, el entrevistador, teniendo en cuenta la impresión subjetiva del paciente, establece un índice de severidad que oscila entre 0 (no existe problema real, el tratamiento no está indicado) y 9 (problema extremo, el tratamiento es absolutamente necesario). En el contexto del EuropASI se define severidad como la necesidad de tratamiento en el caso de que éste no exista o como la implementación de tratamiento adicional. En definitiva, esta entrevista proporciona un perfil con la gravedad que el paciente presenta en cada una de las áreas exploradas.

- International Personality Disorders Examination IPDE- (Loranger, 1994): Es una entrevista semiestructurada, cuyo propósito es identificar rasgos y conductas relevantes para la evaluación de los criterios diagnósticos de los distintos TP según criterios DSM-IV y/o CIE-10. 
El examinador explora la manera de ser habitual del paciente en las siguientes 6 áreas: trabajo, Yo, relaciones interpersonales, afectos, prueba de realidad y control de los impulsos.

Este cuestionario cuenta además con un breve Cuestionario de Evaluación IPDE autoaplicado, de screening, en el que mediante preguntas de respuesta dicotómica (verdadero - falso) el paciente describe su conducta habitual en los últimos 5 años. Este breve cuestionario de screening proporciona al entrevistador una rápida información acerca de qué TP es probable que esté presente y, a continuación la administración del módulo completo del IPDE permitiría confirmar o descartar el diagnóstico de screening.

\section{Procedimiento:}

A todos los pacientes les fueron administrados dichos cuestionarios, en una sesión única, al inicio del estudio, si bien a los pacientes tratados en el centro público se les aplicó la versión DSM-IV del IPDE y a los pacientes del centro privado se les administró la versión CIE-10. De igual modo, a los pacientes privados se les volvió a adimistrar dicho cuestionario a los 6 meses de iniciado el tratamiento (en ambas sesiones fue el mismo investigador el responsable de dicha administración).

\section{Análisis estadístico:}

En el análisis estadístico de las variables cuantitativas se utilizo el test $t$ de Sudent para muestras independientes, mientas que en el análisis estadístico de las variables cualitativas se utilizaron la prueba z de comparación de proporciones (en la comparación de las diferentes submuestras), la prueba chi cuadrado con correción de Yates y el test exacto de Fisher (ambas usadas en las comparaciones realizadas dentro de la misma submuestra). En todos los casos el nivel de significación estadística quedó establecido en el $5 \%$.

\section{RESULTADOS}

\section{Estudio de la muestra basal}

Con el fin de facilitar la comprensión y comparabilidad de los datos, dada la heterogeneidad de procedencia de la muestra hemos decido dividir ésta en tres subgrupos:

PMM público: Compuesta por 53 pacientes sometidos a PMM en el centro público

PMN público: Compuesta por 82 pacientes sometidos a PMN en el centro público

PMN privado: Compuesta por 127 pacientes sometidos a PMN en el centro privado

Características sociodemográficas.- Los pacientes enrolados en PMM público constituyen el grupo de mayor edad [30.52 (6.0) versus 25.50 (4.7) en PMN público, $p=.000$ y versus 27.87 (5.7) en PMN privado, $\mathrm{p}=.007]$, el porcentaje de mujeres es similar en las tres submuestras estudiadas, la submuestra PMN privado tiene un porcentaje de casados superior al de PMN público $(p=.030)$ y el PMN público es el que cuenta con mayor número de sujetos activos (69.5\%), frente a un $52.8 \%$ en el PMN privado $(p=.016)$ y frente a un $50.9 \%$ en el PMM público $(p=.030)$ (ver Tabla 1$)$.

\section{TABLA I: CARACTERISTICAS SOCIODEMOGRAFICASY CLINICAS}

\begin{tabular}{|c|c|c|c|}
\hline & PMM público (n= 53) & PMN público $(n=82)$ & PMN privado $(n=127)$ \\
\hline $\begin{array}{l}\text { Sociodemográficas: } \\
\text { - Edad* } \\
\text { - \% Mujeres** } \\
\text { - \% Casados** } \\
\text { - \% Activos* * }\end{array}$ & $\begin{array}{c}30.52(6.0)^{1,2} \\
22.6 \% \\
15.1 \% \\
50.9 \%^{4}\end{array}$ & $\begin{array}{c}25.50(4.7)^{1,3} \\
14.6 \% \\
12.2 \%{ }^{5} \\
69.5 \%{ }^{4,6}\end{array}$ & $\begin{array}{c}27.87(5.7)^{2,3} \\
15.0 \% \\
24.4 \%{ }^{5} \\
52.8 \%^{6}\end{array}$ \\
\hline $\begin{array}{l}\text { Clínicas: } \\
\text { - Edad inicio* } \\
\text { - i.v. último mes** } \\
\text { - } \mathrm{VHB}^{* *} \\
\text { - } \mathrm{VHC}^{* *} \\
\text { - } \mathrm{VIH}^{* *}\end{array}$ & $\begin{array}{c}21.30(6.0)^{7} \\
67.9 \%^{8,9} \\
47.2 \%^{10,11} \\
73.6 \%^{12,13} \\
20.8 \%^{14,15}\end{array}$ & $\begin{array}{c}19.30(3.3)^{7} \\
32.9 \%^{8} \\
14.6 \%^{10} \\
28.0 \%^{12} \\
0.0 \%^{14}\end{array}$ & $\begin{array}{c}20.43(5.3) \\
22.8 \%^{9} \\
16.5 \%^{11} \\
29.9 \%^{13} \\
3.9 \%^{15}\end{array}$ \\
\hline 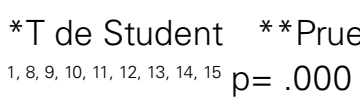 & $\begin{array}{l}\text { a z (comparación de propo } \\
{ }^{2} \mathrm{p}=.007 \quad{ }^{3} \mathrm{p}=.002\end{array}$ & $6,7 p=.016$ & \\
\hline
\end{tabular}


Características clínicas.- El grupo de pacientes en PMN público refieren una edad de inicio de consumo de heroína inferior a la hallada en el PMM público [19.30 (3.3) versus 21.30 (6.0), $p=0.016]$, por otra parte los pacientes tratados en PMM público son los que más han utilizado la vía intravenosa durante el último mes $167.9 \%$ versus un $32.9 \%$ en PMN público, $\mathrm{p}=$ .000 ; y versus un $22.8 \%$ en PMN privado, $p=.000$ ), a la vez que presentan una mayor problemática desde el punto de vista orgánico, como puede deducirse de los porcentajes de seropositividad a VHB $(47.2 \%$ versus $14.6 \%$ en $\mathrm{PMN}$ público, $\mathrm{p}=.000 ; \mathrm{y}$, versus $16.5 \%$ en PMN privado, $p=.000)$ a $\mathrm{VHC}(73.6 \%$ versus $28.0 \%$ en PMN público, $p=.000 ; y$, versus $29.9 \%$ en $P M N$ privado, $p=.000)$ y a $\mathrm{VIH}(20.8 \%$ versus $0 \%$ en $\mathrm{PMN}$ público, $p=.000 ; y$, versus $3.9 \%$ en $P M N$ privado, $p=$ .000). Por otra parte, en las características clínicas descritas no se constata ninguna diferencia entre los PMN público y privado (Tabla 1).

Gravedad de la adicción: EuropASI.- Las áreas donde obtienen mayores puntuaciones todos los pacientes son la de drogas, empleo, familia y relaciones sociales y el área psiquiátrica. No obstante, se observan importantes variaciones de gravedad según la procedencia de los sujetos, de modo, que los pacientes en PMN privado son los tienen puntuaciones de gravedad más bajas en las siete áreas del EuropASI (ver Tabla II), mientras que los pacientes públicos sólo se diferencian en las áreas médica, de drogas y legal, pudiendo constatarse una mayor gravedad en las mencionadas áreas en los pacientes incluidos en PMM [2.73 (2.3) en PMM versus 1.43 (1.3) en PMN, $p=.000$; 7.20 (0.7) en PMM versus 6.92 (0.8) en $P M N, p=.042$; 3.00 (2.4) en PMM versus 2.20 (1.9) en $P M N, p=.036$, respectivamente] (ver Tabla II).

\section{TABLA II: GRAVEDAD DE LA ADICCION: EuropASI}

\begin{tabular}{lccc}
\hline Area EuropASI & PMM público $(n=53)$ & PMN público $(n=82)$ & PMN privado $(n=127)$ \\
\hline Médica & $2.73(2.3)^{1,2}$ & $1.43(1.3)^{1,11}$ & $0.85(1.8)^{2,11}$ \\
Empleo / Soportes & $4.75(2.5)^{3}$ & $4.65(2.3)^{12}$ & $2.37(2.8)^{3,12}$ \\
Alcohol & $1.71(1.0)^{4}$ & $1.75(0.8)^{13}$ & $1.14(1.7)^{4,13}$ \\
Drogas & $7.20(0.7)^{5,6}$ & $6.92(0.8)^{5,14}$ & $3.98(2.5)^{6,14}$ \\
Legal & $3.00(2.4)^{7,8}$ & $2.20(1.9)^{7,15}$ & $1.35(2.2)^{8,15}$ \\
Familiar / Social & $4.00(2.3)^{9}$ & $4.64(2.0)^{16}$ & $2.00(2.4)^{9,16}$ \\
Psiquiátrica & $3.18(2.4)^{10}$ & $2.68(2.1)^{17}$ & $2.00(2.6)^{10,17}$ \\
\hline
\end{tabular}

* T de Student

$1,2,3,6,8,9,12,14,16 p=.000 \quad{ }^{4} p=.027 \quad{ }^{5} p=.042 \quad{ }^{7} p=.036 \quad{ }^{10} p=.006 \quad{ }^{11} p=.014 \quad{ }^{13} p=.003 \quad{ }^{15} p=.005 \quad{ }^{17} p=.049$

Patología dual: IPDE.- El $77 \%$ de pacientes en PMM público presentaron algún tratorno de la personalidad (TP) según criterios IPDE, siendo este porcentaje más elevado que el hallado en la muestra de PMN público $(69 \%, \mathrm{p}=.034)$ y en la de $\mathrm{PMN}$ privado $(42 \%, p=.000)$. De los pacientes tratados en PMN fueron los pertenecientes al centro público los que presentaron mayores porcentajes de comorbilidad $(60 \%$ versus $42 \%, p=.011)$. En la figura 1 puede observarse la distribución del número de TP en cada una de las tres submuestras.

Como puede observarse en la figura 2, los TP más prevalentes en el centro público son el disocial que constituye el $38 \%$ de los diagnósticos en PMM y el $28 \%$ en PMN, el límite $(34 \%$ y $16 \%$ respectivamente), el paranoide (30\% y $10 \%$ respectivamente) y por último, el ansioso (19\% en ambos tipos de tratamiento). En el centro privado el TP más veces diagnosticado ha sido el límite (60\% de los diagnósticos), seguido del disocial (18\%), impulsivo (14\%) y el esquizoide (13\%). En este apartado cabe señalar que cada paciente puede presentar más de un diagnóstico de TP y por otra parte nos gustaría recordar que si bien en la figura 2 se han tratado de aunar los diagnósticos de los diferentes TP, los pacientes públicos han sido evaluados usando versión DSM-IV del IPDE, mientras que en los privados se ha utilizado la versión CIE-10, lo cual puede contribuir en cierto modo a la diferente prevalencia de TP hallada en el centro privado y en el público.

Cuando dentro de las diferentes submuestras tratamos de ver si existe algún parámetro diferencial entre aquellos pacientes que cumplen criterios IPDE de TP y los que no lo cumplen, los hallazgos realizados han sido los siguientes:

PMM público.- Los pacientes que cumplen criterios IPDE de TP (41 sujetos) obtienen puntuaciones de gravedad más bajas en el área médica del EuropASI 
Figura 1. Número de Trastornos de la Personalidad.

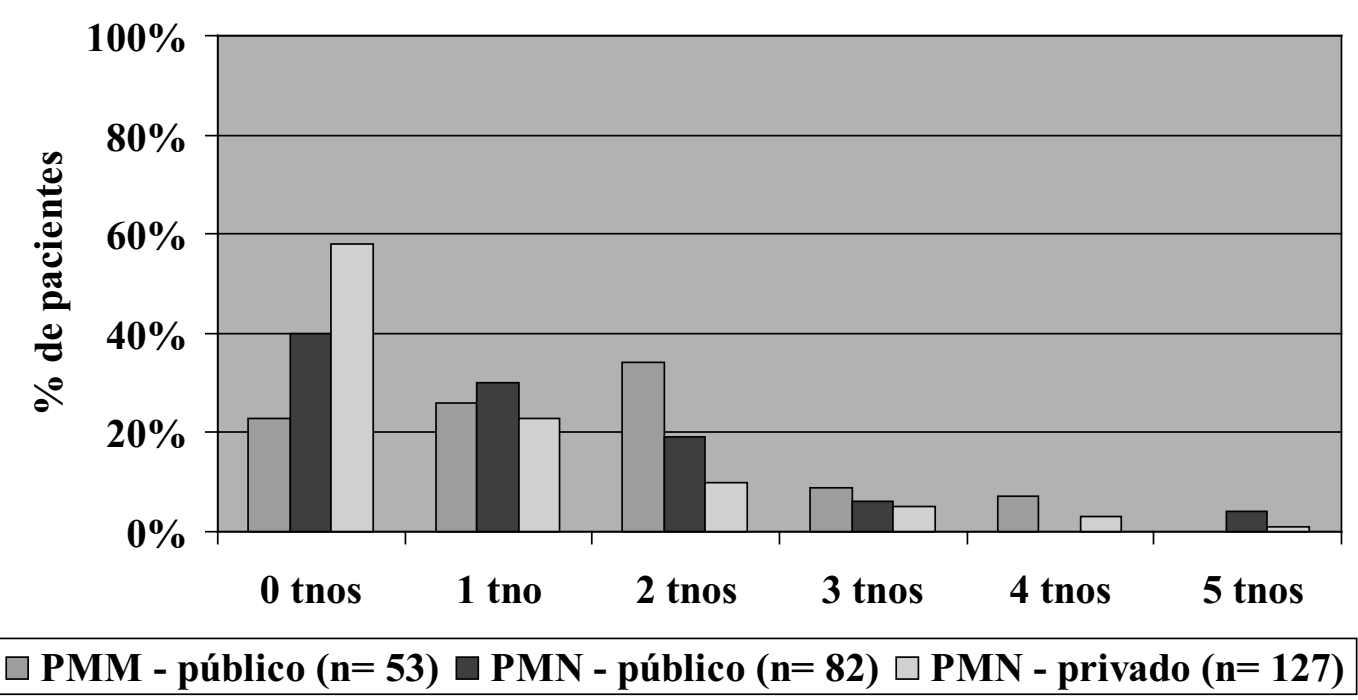

Figura 2. Tipo de Trastorno de la Personalidad

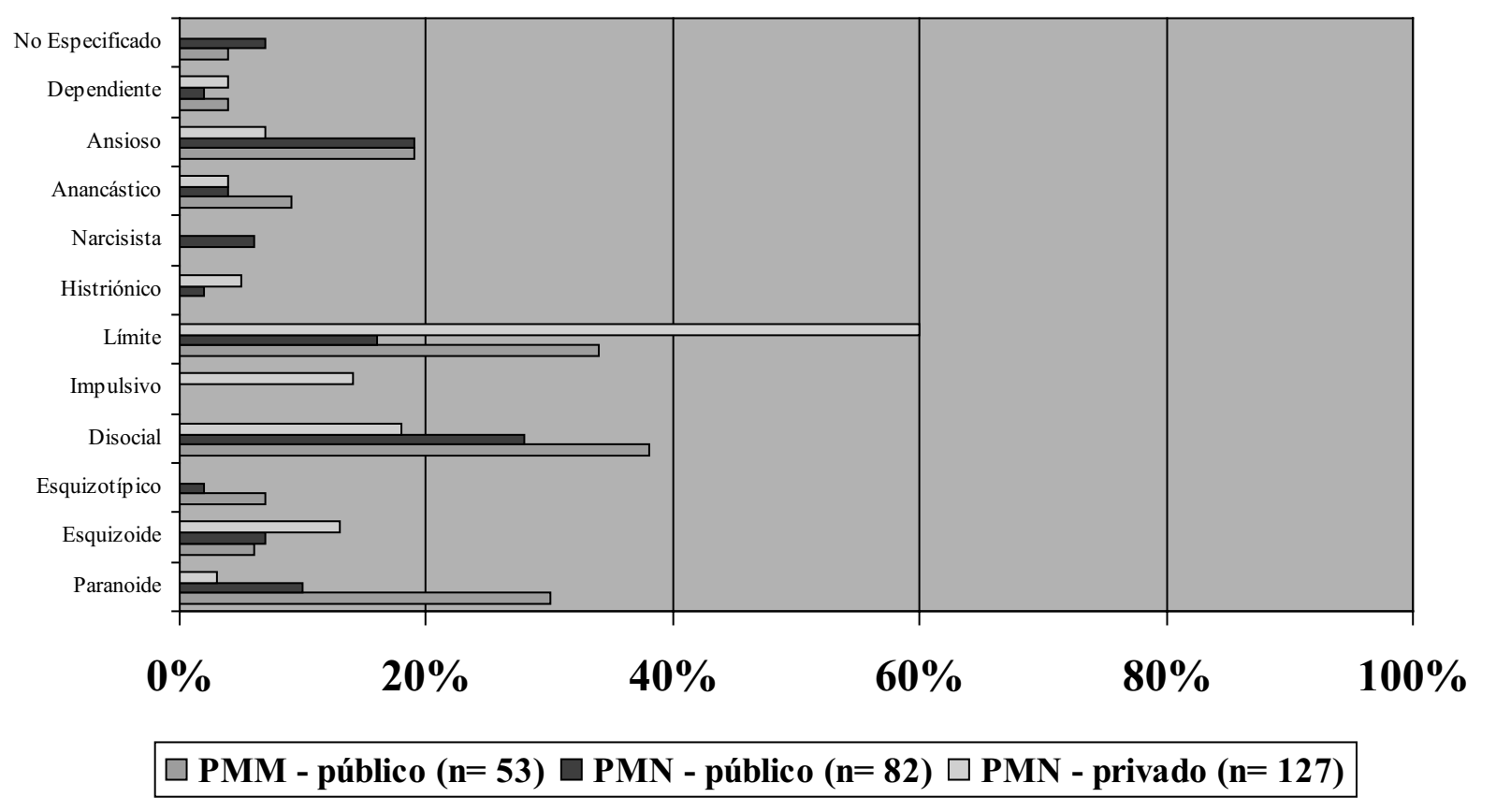

[2.39 (2.0) versus $3.91(2.6), p=.04$ ] y más elevadas en el área de empleo y soportes [5.17 (2.3) versus 3.33 (2.8), $p=.02]$.

PMN público.- Sólo se hallan diferencias estadísticamente significativas en el área de alcohol del EuropASI, con puntuaciones de gravedad superiores para aquellos pacientes con criterios IPDE de TP (49 sujetos) [1.99 (0.9) versus $1.5(0.5), p=.03]$.

PMN privado.- En este subgrupo de pacientes es en el que existen más parámetros diferenciales entre aquellos con criterios IPDE de TP (53 sujetos) y los que no los cumplen. Los pacientes con TP han iniciado más jóvenes el consumo de heroína [18.7 (4.7) años versus 21.6 (5.4) años, $p=.002$ ], han utilizado en mayor proporción la vía intravenosa durante el último mes [34\% versus $15 \%, p=.010]$, y obtienen puntuaciones de gravedad más elevadas en cuatro áreas del EuropASI: empleo y soportes [3.18 (3.0) versus 1.79 (2.5), $\mathrm{p}=.008]$, alcohol [1.66 (1.9) versus $0.77(1.5), p=$ $.008]$, relaciones familiares y sociales [2.6 (2.5) versus $1.66(2.2), p=.020]$ y estado psiquiátrico [3.13 (2.7) versus $1.32(2.3), p=.000]$. 


\section{Estudio de validez del IPDE}

\section{IPDE versión DSM-IV}

En la realización de esta parte del estudio se utilizó una submuestra constituida por 60 de los pacientes que demandaron tratamiento en el centro público. En la determinación de los índices del validez del módulo de screening del IPDE versión DSM-IV se ha utilizado como "gold standard" el módulo completo del IPDE. la versión diagnóstico clínico. El punto de corte establecido a la hora de considerar la presencia de un TP con el screening ha sido 2/3. Se han evaluado por separado cada uno de los TP que contempla la versión DSM-IV, quedando los resultados obtenidos reflejados en la tabla III.

\section{TABLA III: INDICES DE VALIDEZ DEL MODULO DE SCREENING IPDE-DSM-IV}

\begin{tabular}{|c|c|c|c|c|c|}
\hline$T P$ & Sensibilidad & Especificidad & $V P P$ & $V P N$ & Eficiencia \\
\hline Paranoide & $100 \%$ & $41 \%$ & $34 \%$ & $100 \%$ & $55 \%$ \\
\hline Esquizoide & $100 \%$ & $50 \%$ & $24 \%$ & $100 \%$ & $57 \%$ \\
\hline Esquizotípico & $100 \%$ & $54 \%$ & $13 \%$ & $100 \%$ & $57 \%$ \\
\hline Antisocial & $88 \%$ & $64 \%$ & $47 \%$ & $93 \%$ & $70 \%$ \\
\hline Límite & $100 \%$ & $16 \%$ & $28 \%$ & $100 \%$ & $37 \%$ \\
\hline Histriónico & $100 \%$ & $54 \%$ & $4 \%$ & $100 \%$ & $55 \%$ \\
\hline Narcisista & $100 \%$ & $58 \%$ & $11 \%$ & $100 \%$ & $60 \%$ \\
\hline Evitativo & $93 \%$ & $37 \%$ & $31 \%$ & $94 \%$ & $50 \%$ \\
\hline Dependiente & $100 \%$ & $59 \%$ & $8 \%$ & $100 \%$ & $60 \%$ \\
\hline Obsesivo-Compulsivo & $100 \%$ & $76 \%$ & $13 \%$ & $100 \%$ & $77 \%$ \\
\hline No especificado & $0 \%$ & $98 \%$ & $0 \%$ & $93 \%$ & $92 \%$ \\
\hline
\end{tabular}

VPP $=$ Valor Predictivo Positivo $\quad$ VPN= Valor Predictivo Negativo

\section{IPDE versión CIE-10}

La submuestra utilizada para estudiar la validez del módulo de screening del IPDE versión CIE-10 con la versión completa ("gold standard") ha estado constituida, en la visita basal, por los 127 pacientes que consecutivamente acudieron al centro privado y en el seguimiento (visita a los 6 meses) por 35 de dichos pacientes. En este caso no es posible una determinación de los índices de validez del módulo de screening ya que la versión completa sólo se administró a aquellos pacientes que habían resultado positivos en dicho screening. El punto de corte establecido a la hora de considerar la presencia de un TP con el screening ha sido $2 / 3$.

En la visita basal 122 pacientes (96\%) de la muestra obtuvieron puntuaciones superiores a 2 en el screening, es decir presentaban al menos un TP, mientras que tras la administración del módulo completo del IPDE dicho número descendió a 53 (43\% de los positivos en el screening), es decir, 69 sujetos (54\% de la muestra inicial) han resultado ser falsos positivos.

De los 35 pacientes que fueron de nuevo evaluados tras un período de 6 meses, 32 (91\% de los mismos) resultaron de nuevo positivos con el módulo de screening del IPDE, mientras que sólo 8 (25\% de los positivos en el screening) cumplieron diagnóstico de TP según el módulo completo del IPDE, es decir, el número de falsos positivos encontrados en el seguimiento fue de 24 (69\% de la muestra inicial).

Los resultados obtenidos cuando se realiza el mismo análisis teniendo en cuenta los diferentes tipos de TP considerados por la versión CIE-10, tanto en la visita basal como en la visita realizada a los 6 meses, se reflejan respectivamente en las figuras 3 y 4 .

\section{Estudio de seguimiento}

Con el fin de determinar los parámetros relacionados con la retención de los pacientes a medio plazo, éstos fueron sometidos a una segunda evaluación tras 6 meses de tratamiento. Hay que señalar que este estudio sólo se llevó a cabo en la muestra de pacientes que demandaron tratamiento en el centro privado.

De los 127 pacientes que componían la muestra inicial sólo 35 sujetos continuaban en tratamiento a los 6 meses de iniciar el mismo, cifrándose por tanto la retención en un $28 \%$ y el porcentaje de abandonos en un $72 \%$.

Cuando tratamos de establecer el posible perfil diferencial existente entre los pacientes que continuaron tratamiento y los que abandonaron el mismo, observamos que entre ambos grupos no existen diferencias en los parámetros de tipo sociodemográfico 
Figura 3. IPDE versión CIE-10 Visita basal (n=127)

\section{$\square$ Dg. posibles $\square$ Dg. seguros}

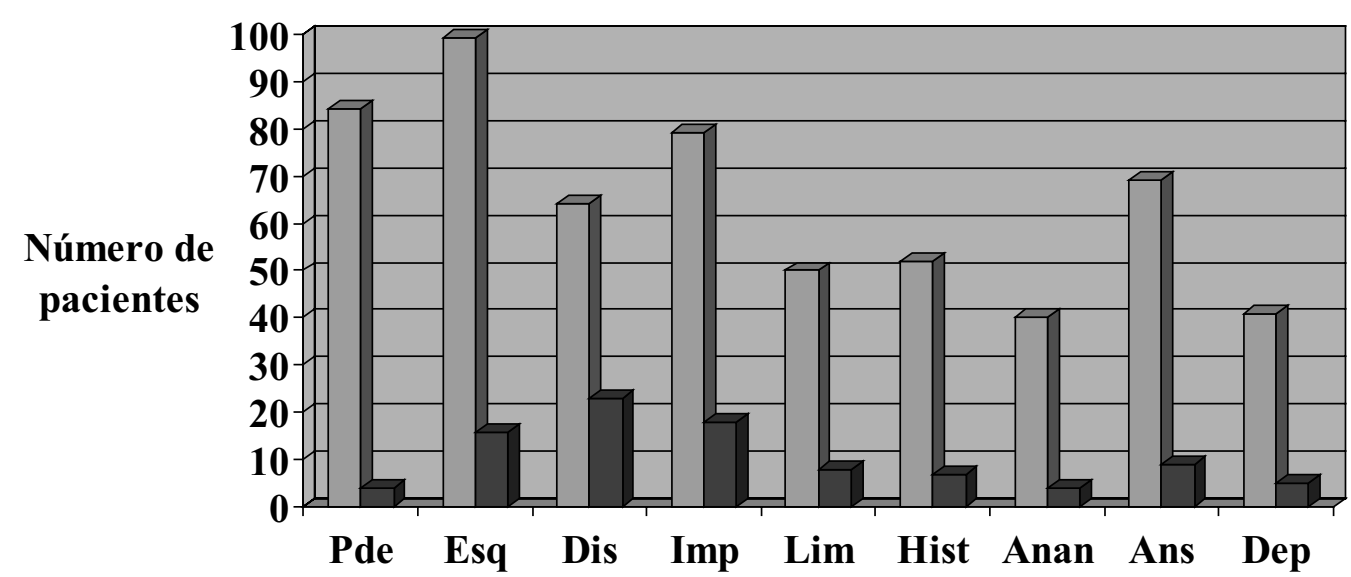

$\begin{array}{llllllllll}\% \text { Falsos }+ & 65 & 75 & 39 & 56 & 35 & 37 & 29 & 51 & 29\end{array}$

\section{Figura 4. IPDE versión CIE-10. Seguimiento -6 meses- (n=35)}

\section{$\square$ Dg. posibles $\square$ Dg. seguros}

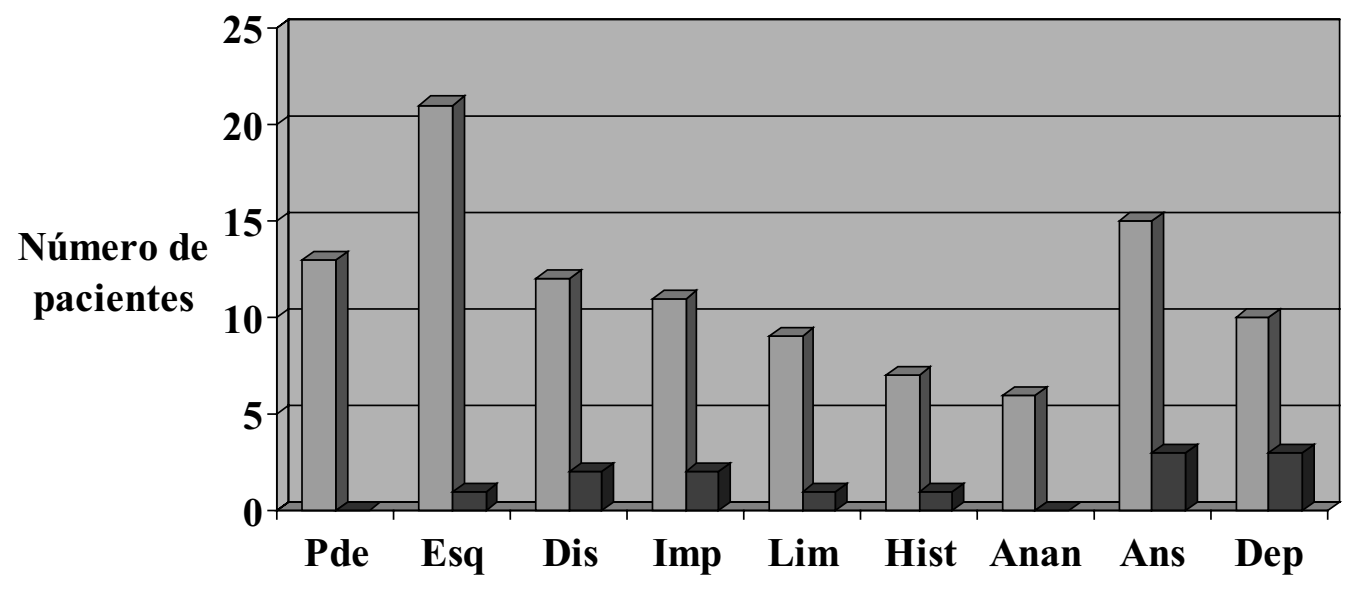

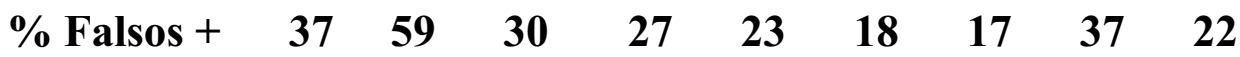

(Tabla IV), mientras que a nivel clínico encontramos que la dosis media inicial de heroína consumida es inferior en el grupo que continúa tratamiento [447.1 (288.7) $\mathrm{mg}$ versus 620.6 (609.4), $\mathrm{p}=$.032] (Tabla IV).

La gravedad de la adicción es mayor en los pacientes que abandonaron el tratamiento antes de los 6 meses, tal y como se deduce del hecho de que éstos obtuvieron puntuaciones medias superiores en tres de las áreas del EuropASI: Alcohol [1.33 (1.89) versus 0.66 (1.39), $p=.032$ ], drogas [4.40 (2.56) versus 2.89
(2.27), $\mathrm{p}=.002]$ y estado psiquiátrico [2.53 (2.80) versus 0.89 (1.86), $p=.000$ ] (Tabla V).

Los pacientes que continuaron tratamiento tenían una media de TP inferior a la de aquellos que abandonaron el mismo [0.40 (0.77) versus 0.87 (1.19), $p=.011]$, aunque prácticamente no se encontraron diferencias en el porcentaje de diagnósticos realizados de los diferentes trastornos en uno y otro grupo, ya que sólo en el caso delTP disocial encontramos porcentajes superiores entre los que abandonaron antes de tiempo el tratamiento [22.8\% versus 5.7\%, $p=.048$ ] (Tabla VI). 
TABLA IV: PARAMETROS DIFERENCIALES: SOCIODEMOGRAFICOS Y CLINICOS

\begin{tabular}{|c|c|c|c|}
\hline & Abandonan $(n=92)$ & Continuan $(n=35)$ & $\mathrm{p}$ \\
\hline Edad $^{1}$ & $28.15(5.94)$ & $27.14(5.20)$ & NS \\
\hline Sexo (varón) $)^{2}$ & $81.5 \%$ & $94.3 \%$ & NS \\
\hline Estado civil (soltero) ${ }^{2}$ & $69.6 \%$ & $57.1 \%$ & NS \\
\hline Situación laboral (activo) ${ }^{2}$ & $46.7 \%$ & $68.6 \%$ & NS \\
\hline Residencia (urbana) ${ }^{3}$ & $88.0 \%$ & $85.7 \%$ & NS \\
\hline Antecedentes Judiciales (juicios) ${ }^{2}$ & $62.0 \%$ & $74.3 \%$ & NS \\
\hline Edad inicio consumo ${ }^{1}$ & $20.38(5.79)$ & $20.66(4.18)$ & NS \\
\hline $\mathrm{VIH}+{ }^{3}$ & $5.4 \%$ & $0.0 \%$ & NS \\
\hline Antecedentes familiares positivos ${ }^{2}$ & $22.8 \%$ & $8.6 \%$ & NS \\
\hline Dosis inicial de heroína' & $620.6(609.4)$ & $447.1(288.7)$ & .032 \\
\hline Meses de abstinencia' & $15.24(26.68)$ & $16.66(39.93)$ & NS \\
\hline $\begin{array}{l}\text { 1T de Student } \\
{ }^{2} \text { Chi cuadrado con correción de Yates } \\
\text { 3Test exacto de Fisher }\end{array}$ & & & \\
\hline
\end{tabular}

\begin{tabular}{|lccc|}
\hline \multicolumn{4}{|c|}{ TABLA V: PARAMETROS DIFERENCIALES: GRAVEDAD DE LA ADICCION } \\
\hline EuropASI & Abandonan $(\mathrm{n}=92)$ & Continuan $(\mathrm{n}=35)$ & $\mathrm{p}$ \\
\hline Médica & $1.00(1.93)$ & $0.49(1.38)$ & $\mathrm{NS}$ \\
Empleo / Soportes & $2.59(2.84)$ & $1.83(2.82)$ & $\mathrm{NS}$ \\
Uso de Alcohol & $1.33(1.89)$ & $0.66(1.39)$ & .032 \\
Uso de Drogas & $4.40(2.56)$ & $2.89(2.27)$ & 002 \\
Situación legal & $1.41(2.24)$ & $1.20(2.25)$ & $\mathrm{NS}$ \\
Relaciones familiares / sociales & $2.25(2.52)$ & $1.54(2.00)$ & $\mathrm{NS}$ \\
Estado psiquiátrico & $2.53(2.80)$ & $0.89(1.86)$ & .000 \\
\hline \multirow{2}{*}{ *T de Student } & & & \\
\hline
\end{tabular}

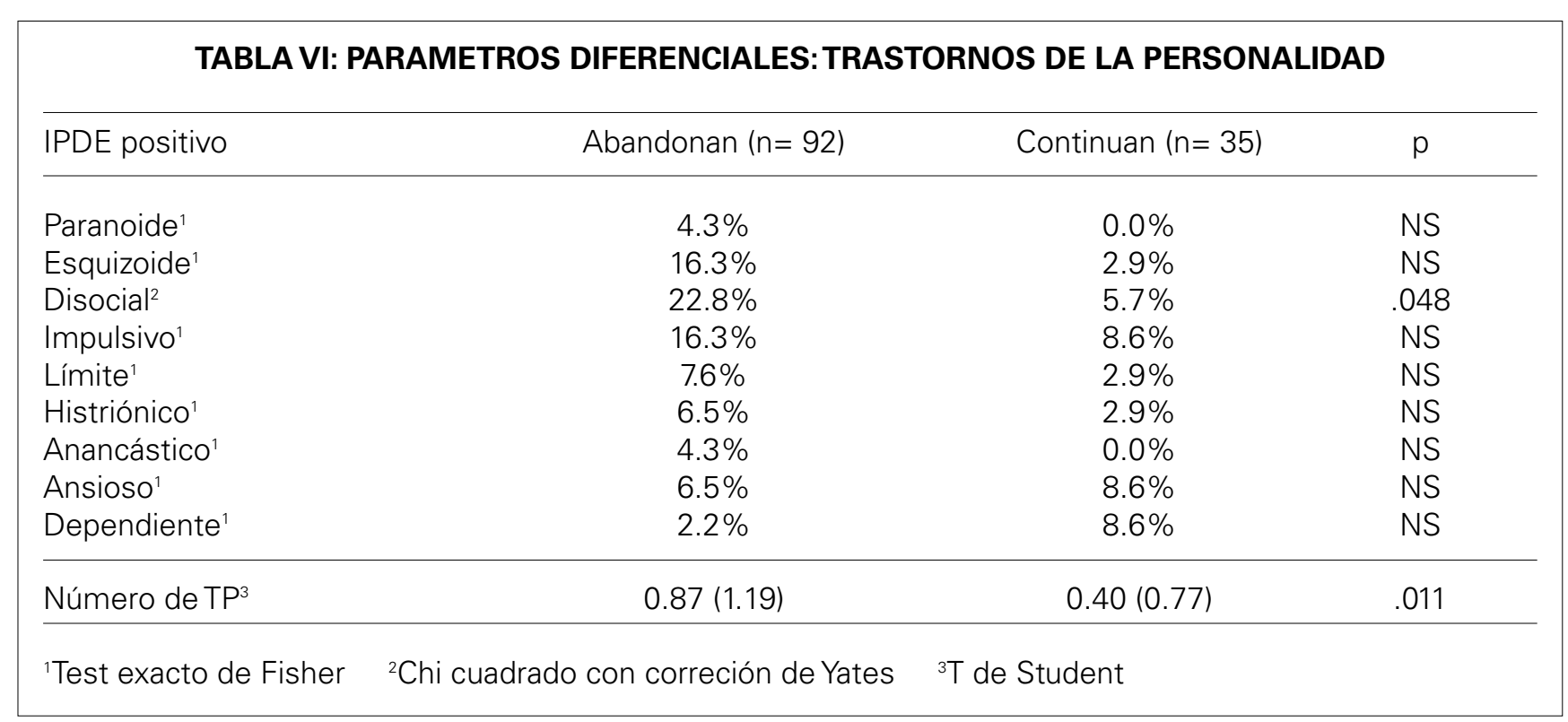

Por último, con el fin de establecer la estabilidad diagnóstica del IPDE repetimos su administración a los 6 meses de iniciado el tratamiento. De los 35 pacientes que se mantuvieron en tratamiento durante 
los 6 meses, 9 cumplieron criterios diagnósticos IPDE en la visita basal: 5 pacientes presentaban un diagnóstico (histriónico, impulsivo, disocial, ansioso y dependiente, respectivamente), 3 pacientes presentaban 2 diagnósticos (ansioso + dependiente, esquizoide + dependiente y disocial + impulsivo) y 1 paciente presentaba 3 diagnósticos de TP (impulsivo + límite + ansioso).

Cuando se les administró de nuevo el cuestionario, tras 6 meses de tratamiento, uno de los pacientes con diagnóstico IPDE de TP en la visita basal, no cumplía criterios diagnósticos en esta segunda visita, concretamente el paciente que había sido diagnosticado de TP impulsivo. Los 26 pacientes que no cumplían criterios diagnósticos de TP en la visita basal siguieron sin cumplirlos en la segunda visita. Dicho de otro modo, podemos cifrar la estabilidad diagnóstica del IPDE a los 6 meses en un 92.8\%, ya que de los 14 diagnósticos realizados inicialmente se mantuvieron 13 , no constatándose ningún nuevo diagnóstico tras ese período.

\section{DISCUSION}

\section{Estudio de la muestra basal}

En lo referente a aspectos sociodemográficos, estos pacientes responden, en líneas generales, a las características observadas en otros estudios realizados en nuestro país (Amodia, 1992; Comas et al, 1992; Marina, 1993; PRD, 1995; Bedate et al, 1995; DGPNSD, 1996; Ruiz et al, 1996; San Narciso et al, 1997, 1998; Cervera et al, 1997; Gutiérrez et al, 1998b).

Al igual que lo descrito por otros autores (Comas et al, 1992; Gómez-Zapata et al, 1992; Marina, 1993; San Narciso et al 1997, 1998; Cervera et al, 1997; Gutiérrez et al, 1998b), los pacientes de la muestra pueden ser considerados como politoxicómanos, aunque el diagnóstico principal sea el de dependencia a opiáceos, ya que han consumido varios tipos de sustancias y actualmente suelen persistir, en su mayoría, en el consumo de diferentes drogas.

De igual modo, la edad media de inicio al consumo de heroína de la muestra es similar ala reportada por los autores anteriormente mencionados. Como cabría esperar la edad y el uso de la vía intravenosa se relacionan claramente con el contagio de enfermedades víricas relacionadas con el consumo de sustancias, siendo los pacientes más veteranos los que más padecen este tipo de problemas ya que hace años, en nuestra Comunidad Autónoma, el uso de la vía intravenosa estaba más extendido entre los consumidores de drogas que en la actualidad (PRD, 1995).
Como en el resto de la Comunidad Autónoma la infección por el VIH es de las más bajas de nuestro entorno y se presenta, sobre todo, en los pacientes de mayor edad, apareciendo pocos casos entre los paciente que más tarde se han incorporado al consumo de drogas, siendo por tanto nuestras cifras más bajas que las referenciadas por autores como Cervera et al (1997) y mucho más acordes con las aparecidas en estudios previos de este grupo de trabajo (San Narciso et al, 1997, 1998; Gutiérrez et al, 1998b).

Por otra parte, y tal y como esperábamos, encontramos que dentro de las tres submuestras incluidas en el presente estudio, son los pacientes sometidos a PMM los que presentan unos criterios de gravedad clínica más acentuados (mayor uso de vía intravenosa durante el último mes y porcentajes más elevados de seroconversión $\mathrm{VHB}, \mathrm{VHC}$ y $\mathrm{VIH}$ ).

La mayor necesidad de tratamiento de nuestros pacientes se centra en las áreas de uso de drogas, de empleo y soportes, de familia y relaciones sociales y en la de estado psiquiátrico. Siendo estos resultados lógicos y compatibles tanto con los problemas derivados de la toxicomanía, con un aumento de la problemática familiar, como con la escasa formación académica de la muetra y los problemas de paro actualmente existentes en nuestra región, así como con las altas tasas de comorbilidad detectadas en el estudio.

El hecho de que los pacientes incluidos en PMM sean los que presenten puntuaciones de gravedad más elevadas, no viene sino a corroborar los criterios clínicos que se tienen en cuenta a la hora de asignar a un paciente a un tipo de tratamiento concreto y el hecho de que los pacientes en PMN público puntúen de manera más elevada que los que estan en PMN privado quizás pueda ser explicado por el hecho de que, en general, los pacientes con mayor problemática y menores recursos económicos y sociales son los que recurren a los centros públicos.

Este estudio subraya la importancia de la patología dual dentro del colectivo de pacientes adictos a opiáceos. Casi las tres cuartas partes de la muestra extraída del centro público (77\% de pacientes en PMM y el $69 \%$ de pacientes en PMN) y cerca de la mitad de la muestra privada (42\%) cumplen criterios IPDE de presentar al menos un TP. Esta discrepancia puede ser debida, en líneas generales, a la mayor gravedad constatada en los pacientes tratados en el centro privado.

La revisión de la literatura arroja que si bien los TP son un diagnóstico muy frecuente en los pacientes adictos a opiáceos, las tasas de comorbilidad son muy dispares oscilando entre un 25-30\% y prácticamente un 90-95\% (Kosten et al, 1982; Khantzian y Treece, 1985; Kosten et al, 1986; García y Ezquiaga, 1992; Rousanville et al, 1992; De Jong et al, 1993; Abbott et al, 1994; Rutherford et al, 1994; Cacciola et al, 1996; 
Ruiz et al, 1996; Brooner et al, 1997; Cervera et al, 1997; San Narciso et al, 1997, 1998; Gutiérrez et al, 1998b, entre otros).

Estas grandes diferencias existentes en lo que a tasas de comorbilidad se refiere, pueden ser debidas a las variaciones metodológicas y poblacionales existentes entre unos estudios y otros. Por ejemplo, existen estudios que realizan la evaluación en el momento de admisión del paciente (Kosten et al, 1982; Khantzian y Treece, 1985), mientras que otros persiguen un período de estabilización de 3 ó 4 semanas (Brooner et al, 1997). Otro problema añadido es el uso de diferentes criterios diagnósticos y el uso de diferentes tipos de entrevistas de ayuda diagnóstica en el terreno de los TP (concretamente con el caso del IPDE, dado que es un instrumento de incorporación muy reciente, hemos encontrado muy pocos estudios, tanto a nivel nacional como internacional, que nos permitan realizar una comparación directa de resultados, con el consiguiente sesgo que este hecho puede generar).

Un nada despreciable porcentaje de los pacientes incluidos es nuestro estudio cumplen criterios de más de un TP, acorde con lo referenciado por otros autores (Nace et al, 1991; De Jong et al, 1993; Weiss et al, 1993; Rutherford et al, 1994; Cervera et al, 1997; San Narciso et al, 1997, 1998; Gutiérrez et al, 1998b). Si bien, el consenso es menor cuando se hace referencia al número medio de TP diagnosticados por persona.

Los TP más frecuentemente diagnosticados en este estudio han sido el antisocial y el límite, lo cual concuerda totalmente con la literatura consultada (Kosten et al, 1982; Khantzian y Treece, 1985; Raskin y Miller, 1993; Abbott et al, 1994; Rutherford et al, 1994; Kokkevi y Stefanis, 1995; Cacciola et al, 1996; Ruiz et al, 1996; Brooner et al, 1997; Cervera et al, 1997; Gutiérrez et al, 1998b), si bien existen trabajos (Miller, 1993; San Narciso et al, 1998) cuyos datos no son concordantes con los expresados con anterioridad. No obstante, es preciso mencionar que en este trabajo, con independencia de la submuestra estudiada, se involucra en mayor o menor medida todo el espectro de TP, lo cual concuerda con lo hallado en estudios previos (Kosten et al, 1982; Rounsaville et al, 1982; Khantzian y Treece, 1985; Abbott et al, 1994; Rutherford et al, 1994; Cacciola et al, 1996; Cervera et al, 1997; Gutiérrez et al, 1998b; San Narciso et al, 1998).

La esperada asociación entre gravedad de la adicción y presencia de TP ha podido ser demostrada, al menos en parte, ya que observamos que los pacientes con criterios IPDE de TP obtienen puntuaciones más elevadas en algunas de las áreas del EuropASI, lo cual puede sin duda ser un factor que ensombrece el pronóstico de estos pacientes (Kosten et al, 1989; Reich y Green, 1991; Brooner et al, 1993; Nace y
Davis, 1993; Perkins et al, 1993; Cacciola et al, 1993; Brooner et al, 1997).

\section{Estudio de validez del IPDE}

Tras realizar el estudio piloto de utilización del IPDE en diferentes culturas y países, sus autores concluyen que es un instrumento dotado de una fiabilidad y validez similar a la de otros instrumentos de uso cotidiano y, quizás la única limitación que encuentran al instrumento, dado que su validación es en el momento actual imposible por no existir un "gold standard" aceptable, resida en el tiempo que se tarda en su administración (Loranger et al, 1994).

La versión de screening de dicho instrumento, el Cuestionario de Evaluación IPDE autoaplicado, constituiría por tanto una herramienta de gran utilidad al clínico si contase con unos índices de validez aceptables. En nuestro estudio, realizado con la versión DSM-IV del cuestionario, hallamos una práctica inexistencia de falsos negativos en todos los TP con excepción del TP no especificado (2 pacientes en el caso de TP antisocial, 1 paciente en el caso del TP por evitación y 4 $-100 \%$ - de los afectados en el caso del TP no especificado). Lo cual trae como consecuencia que la sensibilidad del módulo de screening en todos los tipos de TP, excepto el TP no especificado, sea muy elevada (cifras de 88 a 100\%).

En lo que respecta al número de falsos positivos, éste ha sido más elevado, oscilando entre 38 pacientes en el caso del TP límite y 1 paciente en el caso del TP no especificado, lo cual da lugar a que la especificidad del módulo de screening sea muy variada, oscilando entre el $16 \%$ y $98 \%$.

Dado que el principal requisito que debe de cumplir un instrumento de screening es que esté dotado de una elevada sensibilidad (lo cual se traduciría en la práctica ausencia de falsos negativos) y dado que el TP no especificado es una especie de cajón de sastre donde en ocasiones se incluyen sujetos para los cuales los criterios diagnósticos del resto de TP no están muy claros, consideramos que el módulo de screening constituye una herramienta de gran utilidad en la práctica clínica cotidiana ya que permite la realización de un rápido despistaje de los TP, si bien su baja especificidad obliga a que a la comprobación diagnóstica mediante la utilización de la versión completa del IPDE.

\section{Estudio de seguimiento}

La pérdida de pacientes acaecida durante el periodo de seguimiento puede ser debida, entre otras razones, al hecho de que esos pacientes ya se habían sometido, en la mayoría de los casos, a más de un tratamiento con el fin de abandonar su adicción (concre- 
tamente la media de tratamientos previos realizados era de 3.7), con lo cual consideraban que las modalidades de tratamiento de toxicomanías existentes en el momento actual no eran válidas para ellos, puesto que habían recaído y por tanto llegaban a este nuevo proceso terapeútico mermados en su motivación. Por otra parte, no hay que olvidar que esta parte del estudio se realizó con los pacientes que estaban en tratamiento en el centro privado, por lo que el coste económico del mismo puede ser otro condicionante que hay que tener presente a la hora de explicar la baja adherencia terapeútica.

Cuando tratamos de establecer las posibles diferencias existentes entre aquellos pacientes que abandonan el tratamiento y los que permanecen en el mismo durante un período de al menos 6 meses, hallamos que si bien no existen muchos parámetros diferenciales, el grupo de los que abandonan presentan una mayor gravedad clínica y psicológica que se traduce en mayores dosis iniciales de consumo de heroína, mayor severidad en el uso de alcohol y drogas y en su estado psiquiátrico, con una mayor media de TP diagnosticados. Factores todos ellos que sin duda pueden tener mucho que ver en el pronóstico a medio y largo plazo de estos pacientes.

EI IPDE es un cuestionario que evalúa rasgos o comportamientos estables del sujeto, entendiendo por estabilidad un mínimo de 5 años, incluyendo alguna manifestación de su comportamiento durante los últimos 12 meses. Dado que en nuestro estudio los pacientes fueron reexaminados al cabo de 6 meses, se requeriría para una adecuada estabilidad diagnóstica del cuestionario, que la administración del instrumento proveyera al examinador una información similar que la obtenida en la primera ocasión (visita basal), si bien la existencia de pacientes que tras los 6 meses cumpliesen ya el criterio de 5 años (y pasaran por tanto a ser diagnosticados) o pacientes que ya no cumpliesen el criterio "alguna manifestación de su comportamiento durante los últimos 12 meses" (y, por tanto dejaran de ser diagnosticados) podría afectar a la estabilidad temporal del instrumento. La estabilidad temporal del instrumento puede, asimismo, verse afectada por la pérdida de fiabilidad derivada de una única administración del instrumento tanto en la visita basal como en la de seguimiento (Loranger et al, 1994).

En la actualidad existen escasos estudios que informen acerca de la estabilidad temporal de la mayoría de la entrevistas semiestructuradas que son usualmente utilizadas para el diagnóstico de trastornos psicóticos, afectivos, de ansiedad y de uso-abuso de sustancias (Andreasen et al, 1981; Bromet et al, 1986; Rice et al, 1986, 1992; Fendrich et al, 1990). Dichos estudios reflejan una moderada, aunque a veces decepcionante, estabilidad temporal no superior a la encontrada en el estudio realizado por Loranger et al (1994), con el IPDE.

De igual modo también son muy escasos los estudios realizados para establecer la estabilidad temporal de entrevistas semiestructuradas de utilidad diagnóstica en los TP (Van den Brink, 1989; Loranger et al, 1991; Pfohl et al, 1991) y, de igual modo, los resultados obtenidos son bastante similares a los encontrados por Loranger et al (1994) con el IPDE.

En nuestro estudio solamente encontramos un caso (con criterios de TP impulsivo), en el que no fue posible refrendar el diagnóstico a los 6 meses, lo cual nos sirve para refrendar desde nuestra práctica clínica una adecuada estabilidad temporal, tal y como habían previamente descrito Loranger et al (1994).

\section{CONCLUSIONES}

A pesar de las limitaciones metodológicas que pudieran presentar los trabajos realizados, los resultados expuestos en las líneas precedentes nos permiten realizar las siguientes conclusiones:

- La prevalencia de TP es mayor en los pacientes sometidos a Programa de Mantenimiento con Metadona

- La distribución de TP varía en función de la modalidad terapeútica estudiada, así en Programa de Mantenimiento con Metadona público los TP más frecuentemente diagnosticados son el disocial, el límite y el paranoide, en Programa de Mantenimiento con Naltrexona público el disocial, ansioso y límite, y, por último en Programa de Mantenimiento con Naltrexona privado el límite, disocial e impulsivo.

- En líneas generales, puede decirse que los adictos a opiáceos que cumplen criterios IPDE de TP presentan una mayor gravedad de su adicción, gravedad que se manifiesta por puntuaciones más elevadas en algunas áreas del EuropASI

- El módulo de screening del IPDE ha demostrado tener una elevada sensibilidad y moderada especificidad.

- Los TP con mayor índice de falsos positivos cuando se usa el módulo de screening IPDE-DSM-IV han sido el límite, el evitativo, el paranoide y el histriónico, mientras que cuando se utiliza el módulo CIE-10 resultaron ser el esquizoide, el paranoide y el impulsivo.

- Los pacientes que abandonan precozmente el tratamiento en PMN privado presentan un perfil caracterizado por una mayor dosis inicial de heroína consumida, una mayor gravedad de la adicción que se manifiesta por unas puntuaciones más elevadas en algunas áreas del EuropASI 
(alcohol, drogas y estado psiquiátrico) y, por último, un mayor número de diagnósticos IPDE de TP.

- Queda confirmada la estabilidad diagnóstica del IPDE a medio plazo (6 meses).

\section{BIBLIOGRAFIA}

Abbott PJ, Weller SB, Walker SR (1994): Psychiatric disorders of opiod addicts entering treatment: Preliminary data. J Addict Dis, 13 (3): 1-11.

Alterman Al, Cacciola JS (1991): The antisocial personality disorder in substance abusers: Problems and issues. $\mathbf{J}$ Nerv Ment Dis, 179: 401-409.

Amodia Díez R (1992): Epidemiología de las toxicomanías en la red de salud mental de Asturias (1980-1989). Cuadernos Asturianos de Salud. Oviedo, Servicio de Publicaciones del Principado de Asturias.

Andreasen NC, Grove WM, Shapiro RW et al (1981): Reliability of lifetime diagnosis: A multicenter collaborative perspective. Arch Gen Psychiatry, 38: 400-405.

Bedate J, Bobes J, Ochoa E, Solé J (1995): Evaluación del tratamiento de los heroinómanos con naltrexona. Estudio multicéntrico. En: Casas M, Gutiérrez M, San L (eds.), Avances en drogodependencias. Barcelona, Ediciones en Neurociencias.

Bobes J, Bousoño M, González MP et al (1994): Aspectos psicopatológicos y familiares del drogodependiente en la Comunidad Autónoma del Principado de Asturias. Libro de Actas del Congreso Nacional de Psiquiatría. Zaragoza.

Bromet EJ, Dunn LO, Connell MM et al (1986): Long-term reliability of diagnosing lifetime major depression in a community sample. Arch Gen Psychiatry, 43: 435440.

Brooner RK, Greenfield L, Schmidt CW (1993): Antisocial personality disorders and HIV infection among intravenous drug abusers. Am J Psychiatry, 150: 53-58.

Brooner RK, King VL, Kidorf M et al (1997): Psychiatric and substance use comorbidity among treatment-seeking opioid abusers. Arch Gen Psychiatry, 54: 71-80.

Cacciola JS, Rutherford MJ, Alterman Al et al (1996): Personality disorders and treatment outcome in methadone maintenance patients. J Nerv Ment Dis, 184 (4): 234239.

Carey MP, Carey KB, Meisler AW (1991): Psychiatric symptoms in mentally ill chemical abusers. J Nerv Ment Dis, 179: 136-138.

Cervera G, Valderrama JC, Bolinches F, Martínez J (1997): Pauta de desintoxicación frente a estabilización y mantenimiento con metadona en adictos a opiáceos con trastorno de la personalidad. Psiquiatría Biológica, 4 (5): 181-186.
Comas D, Carrón J, Hernández MI et al (1992): Metodología de los estudios de seguimiento. Resultados de una investigación. Adicciones, 4 (2): 111-126.

De Jong CAJ, Van der Brink W, Harteveld FH et al (1993): Personality disorders in alcoholics and drug addicts. Compr Psychiatry, 34 (2): 87-94.

Delegación del Gobierno para el Plan Nacional sobre Drogas (1996): Sistema estatal de información sobre toxicomanías (S.E.I.T.) 1995. Madrid, Ministerio de Justicia e Interior.

Fendrich M, Weissman MM, Warner V, Mufson L (1990): Two-year recall of lifetime diagnoses in offspring at high and low risk for major depression: The stability of offspring reports. Arch Gen Psychiatry, 47: 1121-1127.

García López A, Ezquiaga Terrazas E (1992): Psicopatología asociada al consumo de drogas. Actas Luso-Esp Neurol Psiquiatr, 20 (4): 175-187.

Gerstley LJ, Alterman AI, McLellan AT et al (1990): Antisocial personality disorder in patients with substance abuse disorders: A problematic diagnosis?. Am J Psychiatry, 147 (2): 173-178.

Gómez Zapata M, Osuna E, Seiquer C et al (1992): Patología orgánica asociada en adictos a drogas por vía parenteral. Estudio de una muestra de 200 pacientes. Adicciones, 4 (2): 127-124.

Gutiérrez E, Sáiz PA, González MP et al (1998a): Trastornos de la personalidad en adictos a opiáceos en tratamiento con agonistas vs antagonistas. Adicciones, 10 (2): 121-129.

Gutiérrez E, Sáiz PA, González MP et al (1998b): Definición del perfil de buen respondedor a los programas de mantenimiento con naltrexona. Adicciones, 1: 321333.

Khantzian EJ, Treece K (1985): DSM-III psychiatric diagnosis of narcotic addicts: Recent findings. Arch Gen Psychiatry, 45: 1067-1071.

Kokkevi A, Hartgers C: EuropASI (1995): European Adaptation of a multidimensional assessment instrument for drug and alcohol dependence. Eur Addict Res, 1: 208210.

Kokkevi A, Stefanis C (1995): Drug abuse and psychiatric comorbidity. Compr Psychiatry, 36 (5): 329-337.

Kosten TR, Rounsaville BJ, Kleber HD (1982): DSM-III personality disorders in opiate addicts. Compr Psychiatry, 23: 572-581.

Kosten TR, Rousanville BJ (1986): Psychopathology in opioid addicts. Psychiatric Clinics of North America, 9 (3): 515-532.

Kosten TA, Kosten TR, Rounsaville BJ (1989): Personality disorders in opiate addicts prognostic specificity. $\mathbf{J}$ Subst Abuse Treat, 6: 163-168.

Loranger AW, Lenzenweger MF, Gartner AF et al (1991): Trait-state artifacts and the diagnosis of personality disorders. Arch Gen Psychiatry, 48: 720-728.

Loranger AW, Sartorius N, Andreoli A et al (1994): The International Personality Disorder Examination. Arch Gen Psychiatry, 51: 215-224. 
Marina P (1993): Adictos a la heroína en Asturias. Un estudio de seguimiento. Cuadernos Asturianos de Salud. Oviedo, Servicio de Publicaciones del Principado de Asturias.

McLellan AT (1986): Psychiatric severity as a predictor of outcome from substance abuse treatment. En: Meyer RE (ed.), Psychopathology and addictive disorders. New York, The Guilford Press.

Miller NS (1993): Comorbidity of psychiatric and alcohol/drug disorders: Interactions and independent status. J Addict Dis, 12 (3): 5-16.

Nace EP, Davis CW, Gaspari J (1991): Axis II comorbidity in substance abusers. Am J Psychiatry, 148: 118-120.

Nace EP, Davis CW (1993): Treatment outcome in substance abusing patients with personality disorder. Am J Addict, 2 (1): 26-33.

Perkins DO, Davidson EJ, Leserman J et al (1993): Personality disorders in patients infected with HIV: A controlled study. Am J Psychiatry, 150 (2): 309-315.

Pettinati HM, Jensen JM, Tracy JI et al (1991): Cocaine vs alcohol dependence: Axis II and outcome. En: Alterman AL (ed.), Research Society on Alcoholism. FL, Marco Island.

Pfohl B, Black DW, Noyes R et al (1991): Axis I and Axis II comorbidity findings: Implications for validity. En: Oldham JM (ed.), Personality disorders: New perspectives on diagnostic validity. Washington $D C$, American Psychiatric Press.

Plan Regional sobre Drogas (1995): Memoria 1994. Oviedo, Servicio de Publicaciones del Principado de Asturias.

Randval E, Vaglum P (1991a): Changes in antisocial aggressiveness during treatment in a hierarchical therapeutic community. A prospective study of personality changes. Acta Psychiatr Scand, 84 (6): 524-530.

Randval E, Vaglum P (1991b): Psychopathology and substance abuse as predictor of program completion in a therapeutic community for drug abusers: A prospective study. Acta Psychiatr Scand, 83 (3): 217-222.

Raskin VD, Miller NS (1993): The epidemiology of the comorbidity of psychiatric and addictive disorders: A critical review. J Addict Dis, 12 (3): 45-57.
Regier DA, Kaelber CT, Rae DS et al (1998): Limitations of diagnostic criteria and assessment instruments for mental disorders. Arch Gen Psychiatry, 55: 109-115.

Reich JH, Green Al (1991): Effect of personality disorders on outcome of treatment. J Nerv Ment Dis, 179: 74-82.

Rice JP, McDonald-Scott P, Endicott J et al (1986): The stability of diagnosis with an application to bipolar II disorder. Psychiatry Res, 19: 285-296.

Rice JP, Rochberg N, Endicott J et al (1992): Stability of psychiatric diagnoses: An application to the affective disorders. Arch Gen Psychiatry, 49: 824-830.

Rounsaville BJ, Weissman MM, Kleber H et al (1982): Heterogeneity of psychiatric diagnosis in treated opiate addicts. Arch Gen Psychiatric, 39: 161-166.

Rousar E, Brooner RK, Reiger MW et al (1994): Psychiatric distress in antisocial drug abusers: Relation to other personality disorders. Drug Alcohol Depend, 34: 149154.

Ruiz R, Casete L, Carrera IH et al (1996): Estudio preliminar sobre la incidencia y valor pronóstico del codiagnóstico psiquiátrico en usuarios de una comunidad terapeútica. Libro de Actas de las XXIII Jornadas Nacionales de Socidrogalcohol, Oviedo.

Rutherford MJ, Cacciola JS, Alterman Al (1994): Relationships of personality disorders with problem severity in methadone patients. Drug Alcohol Depend, 35: 69-76.

San Narciso GI, Carreño JE, Pérez SF et al (1998): Evolución de los trastornos de personalidad evaluados mediante el IPDE en una muestra de pacientes heroinómanos en tratamiento con naltrexona. Adicciones, 10 (1): 7-21.

Tyrer P (1988): What's wrong with DSM-III personality disorders?. J Pers Disord, 2: 281-291.

Van den Brink W (1989): Meting van DSM-III persoonlijkheidspathologie: Betrouwbaarheid en validiteit van de SIDP-R as II van de DSM-III. Groningen, Drukkerij Van Derenden BV.

Weiss RD, Mirin SM, Griffin ML et al (1993): Personality disorders in cocaine dependence. Compr Psychiatry, 34: $145-149$. 